\title{
Comparison of Treatment Effects Between Children With Spastic Cerebral Palsy Under and Over Five Years After Botulinum Toxin Type A Injection
}

\author{
Won-Yub Lee, MD, Gi-Young Park, MD, Dong Rak Kwon, MD
}

Department of Rehabilitation Medicine, Catholic University of Daegu School of Medicine, Daegu, Korea

\begin{abstract}
Objective To evaluate whether age influences a change in the spasticity of the ankle plantar flexor after botulinum toxin type A (BTA) injection in children with spastic cerebral palsy (CP).

Methods Sixteen children with spastic CP were enrolled in the study. Seven children (group 1) were under 5 years of age, and nine (group 2) were over 5 years of age. They all received BTA injection in the gastrocnemius muscle (GCM) under ultrasound guidance. Passive range of motion (PROM) of ankle dorsiflexion, Modified Ashworth Scale (MAS) of the ankle plantar flexor, Gross Motor Function Measure (GMFM) and median red pixel intensity (RPI) of the medial GCM on real-time sonoelastography were measured at baseline (pre-injection) and 1-, 3-, and 6-month post-injection.

Results In both groups, the mean PROM, MAS, and RPI were significantly improved after injection until 6-month post-injection. The change of PROM of ankle dorsiflexion in group 1 was significantly greater than that in group 2 , until 6-month post-injection. The change in the MAS and GMFM between baseline and 6-month post-injection in group 1 was greater than that in group 2. The changes in the median RPI between baseline and 3- and 6-month post-injections were greater in group 1 than in group 2.

Conclusion Our pilot study demonstrated the different changes in spasticity of the ankle plantar flexor after BTA injection based on age. Therefore, age may be considered when establishing a treatment plan using BTA injection for children with spastic CP.
\end{abstract}

Keywords Cerebral palsy, Age, Muscle spasticity, Botulinum toxin type A, Sonoelastography

Received May 9, 2013; Accepted September 26, 2013

Corresponding author: Dong Rak Kwon

Department of Rehabilitation Medicine, Catholic University of Daegu School of Medicine, 33 Duryugongwon-ro 17-gil, Nam-gu, Daegu 705718, Korea

Tel: +82-53-650-4878, Fax: +82-53-622-4687, E-mail: coolkwon@cu.ac.kr

(c) This is an open-access article distributed under the terms of the Creative Commons Attribution Non-Commercial License (http://creativecommons. org/licenses/by-nc/3.0) which permits unrestricted noncommercial use, distribution, and reproduction in any medium, provided the original work is properly cited.

Copyright $\odot 2014$ by Korean Academy of Rehabilitation Medicine

\section{INTRODUCTION}

Cerebral palsy (CP) is a non-progressive neurological illness presenting with multiple impairments, including spasticity, weakness, and impaired motor control. This neurological disorder can cause secondary alterations in the musculoskeletal system, such as decreased muscle strength and tightness or contractures around the joints. 
Spasticity is defined as a velocity-dependent resistance to passive stretch and is clinically assessed using the Modified Ashworth Scale (MAS) and the Tardieu scale $[1,2]$. Spasticity in clinical testing reflects a combination of intrinsic (non-neuronal component) and reflex-mediated (neuronal component) muscle stiffness. Secondary changes in intrinsic muscle property, such as collagen and tendon properties (e.g., loss of sarcomeres and subclinical contractures) result in spastic muscle tone, which in part compensates for paresis [3]. Collagen synthesis of the spastic muscle in CP may be stimulated directly through exposure of the spastic muscle to abnormal mechanical loads, which result from an abnormal neuronal firing pattern [4].

Local injection of botulinum toxin type A (BTA) into spastic muscles results in reversible disruption of acetylcholine release from the muscle's nerve endings $[5,6]$, enabling increased function over time [7]. The use of BTA as an adjunct to other interventions, including physiotherapy and casting, has been recommended for the management of children with spastic CP in order to reduce spasticity, prevent or delay orthopedic surgery and improve function and gait $[6,8]$.

Real-time sonoelastography (RTS) is a recently developed ultrasound-based imaging technique that evaluates tissue elasticity in real-time. RTS is in accordance with the principle that tissue compression produces strain (displacement), which is low in hard tissues and high in soft tissues [9]. RTS demonstrated a difference in intrinsic muscle stiffness between spastic and normal gastrocnemius muscles (GCMs) in children with CP [10]. The increased collagen content in the spastic muscle is indirectly estimated using RTS, since RTS measures intrinsic muscle stiffness. In children with spastic $\mathrm{CP}$, changes in intrinsic muscle stiffness after BTA injection have been documented using RTS $[11,12]$. Intrinsic muscle stiffness increases with age-related rise of non-reducible collagen content in spastic muscle [13]. A previous study has claimed that as the age of children with spastic CP increases, passive intrinsic muscle stiffness may play a more important role in spasticity than do reflex-mediated activity [14].

We investigated the difference of changes of the ankle plantar flexor spasticity using MAS, intrinsic muscle stiffness using passive range of motion (PROM) and RTS and functional outcome using Gross Motor Function Measure
(GMFM), after BTA injection in children with spastic CP, according to age.

\section{MATERIALS AND METHODS}

\section{Participants}

Sixteen children (mean age, 6.0 \pm 2.7 years [range, 2.3-9.5 years]), including 10 with diplegia and 6 with hemiplegia, were admitted to the inpatient clinic of the Department of Rehabilitation Medicine at Daegu Catholic University Medical Center. Group 1 included 7 children (3 boys and 4 girls; age, $3.7 \pm 1.1$ years; 4 with diplegia and 3 with hemiplegia, 11 legs; mean body weight, $14.2 \mathrm{~kg}$ [range, 10-21 kg]; 2 with Gross Motor Function Classification System [GMFCS] level II and 5 with GMFCS level III) under 5 years of age. Group 2 included 9 children ( 5 boys and 4 girls; age, $9.2 \pm 1.7$ years; 6 with diplegia and 3 with hemiplegia, 15 legs; mean body weight, $27.5 \mathrm{~kg}$ [range, 19-42 kg]; 1 with GMFCS level II and 8 with GMFCS level III) over 5 years of age. The inclusion criteria were as follows: 1) verified diagnosis of $\mathrm{CP}$ by a physiatrist specialized in pediatric rehabilitation medicine; 2) independent ambulation with or without gait-assistive devices; 3 ) spastic gait with equinus of the ankle; and 4) dynamic ankle contracture. Dynamic ankle contracture was confirmed if equinus of the ankle was observed during ambulation, and passive dorsiflexion of the ankle could be accomplished beyond the neutral position with the knee extended. Exclusion criteria were as follows: 1) more than 13 years or less than 2 years of age; 2) previous BTA injection in the GCM or serial casting of the ankle within 6 months before enrolment; 3) fixed ankle contracture; and 4) surgery of the lower limbs in the 12 months prior to inclusion. The Institutional Review Board and Ethics Committee at Daegu Catholic University Medical Center approved the protocol of this study. Because all the children in this study were younger than 18 years of age, informed consent for participation was obtained from the parents of the children.

\section{Intervention}

All the children received the same intervention, including rehabilitation treatment and an intramuscular injection of BTA (Meditoxin; Medytox Inc., Seoul, Korea) in each medial and lateral GCM muscle under ultrasound guidance. The BTA dosage was calculated in accordance 
with the current guidelines accessible at the time of treatment [15]: the dose for hemiplegic and diplegic CP children was $4 \mathrm{U} / \mathrm{kg}$ and $6 \mathrm{U} / \mathrm{kg}$, respectively. No adverse effects or complications were observed after the injection. Before the injection, all the children underwent outpatient rehabilitation treatment once a week. After the injection, inpatient rehabilitation treatment, including stretching and strengthening exercises, functional electrical stimulation and progressive gait training, was performed twice daily for four weeks. After the inpatient rehabilitation treatment, the frequency for outpatient rehabilitation treatment for all the children was twice a week for six months.

\section{Clinical measurements}

Clinical assessments were performed at baseline (preinjection) and 1-, 3-, and 6-month post-injections. PROM of ankle dorsiflexion was assessed using a classical twoarm goniometer in the supine position, with the knee fully extended and knee flexed to $90^{\circ}$ by the physiatrist throughout the study. Spasticity of the ankle plantar flexor was measured as the degree of resistance to passive movement, using the MAS, and was rated on a scale of 0 (normal) to 5 (extreme), according to the amount of resistance felt by the physical therapist. The physical therapist also measured the GMFM.

\section{RTS}

Ultrasound and RTS of the medial GCM were performed simultaneously using a commercially available ultrasound system with a 5- to $13-\mathrm{MHz}$ multifrequency linear transducer (Antares; Siemens Healthcare, Erlangen, Germany) by a physiatrist (17 years of experience in musculoskeletal ultrasound and 4 years of experience in RTS), at baseline and 1-, 3-, and 6-month post-injections. Children were scanned on the longitudinal plane of the medial GCM, and natural compression was applied with the transducer in accordance with normal respiration. All the children were scanned on an examination table in the prone position with their feet hanging off the edge. Scanning was discontinued whenever reflexive or voluntary contraction of the lower limb muscles was observed. The proximal tendon, musculotendinous junction and muscle portion of the medial GCM were easily recognized from proximal to distal scanning of the leg on ultrasound. Scanning was performed repeatedly at a fixed point on the medial GCM, which was located between two reference points: one point was located at the proximal onethird of a longitudinal line from midway between the medial and lateral malleoli to midway between the medial and lateral epicondyles; and the other point was located at the medial end on a transverse line, perpendicular to the point on the longitudinal line. Using ultrasound guidance, a region of interest (ROI) was selected, which included the medial GCM fascicles with clearly demarcated

Table 1. Comparison of changes in the passive range of motion (PROM) of the ankle at baseline and post-injection between group 1 and group 2

\begin{tabular}{|c|c|c|c|c|}
\hline & \multicolumn{2}{|c|}{ PROM $\left({ }^{\circ}\right)$ with KE } & \multicolumn{2}{|c|}{ PROM $\left({ }^{\circ}\right)$ with KF } \\
\hline & Group $1(n=11)$ & Group $2(n=15)$ & Group $1(n=11)$ & Group $2(n=15)$ \\
\hline Baseline & $6.3 \pm 2.3$ & $5.7 \pm 1.5$ & $19.5 \pm 2.2$ & $18.0 \pm 2.1$ \\
\hline 1MPI & $19.5 \pm 4.1^{\mathrm{a}, \mathrm{b})}$ & $15.3 \pm 4.4^{\mathrm{b})}$ & $34.1 \pm 3.5^{\mathrm{a}, \mathrm{b})}$ & $28.1 \pm 3.0^{\mathrm{b})}$ \\
\hline$\Delta$ & $13.1 \pm 4.6^{\mathrm{a})}$ & $9.6 \pm 4.6$ & $14.6 \pm 3.3^{\mathrm{a})}$ & $10.1 \pm 3.5$ \\
\hline 3MPI & $19.6 \pm 0.8^{\mathrm{a}, \mathrm{b})}$ & $12.0 \pm 4.1^{\mathrm{b})}$ & $33.6 \pm 5.9^{\mathrm{a}, \mathrm{b})}$ & $22.6 \pm 4.5^{\mathrm{b})}$ \\
\hline$\Delta$ & $13.2 \pm 2.6^{\mathrm{a})}$ & $6.2 \pm 3.6$ & $14.0 \pm 5.6^{\mathrm{a})}$ & $4.6 \pm 4.7$ \\
\hline 6MPI & $16.8 \pm 2.5^{\mathrm{a}, \mathrm{b})}$ & $7.2 \pm 2.4^{\mathrm{b})}$ & $31.3 \pm 5.0^{\mathrm{a}, \mathrm{b})}$ & $19.6 \pm 2.9^{b)}$ \\
\hline$\Delta$ & $10.4 \pm 3.5^{\mathrm{a})}$ & $1.4 \pm 2.2$ & $11.8 \pm 5.0^{\mathrm{a})}$ & $1.6 \pm 2.3$ \\
\hline
\end{tabular}

Values are presented as mean \pm standard deviation.

PROM, passive range of motion; KE, knee extension; KF, knee flexion; MPI, month post-injection; $\Delta$, difference in the mean degrees between baseline and at each month post-injection; group 1, under 5 years of age; group 2, over 5 years of age.

a) $\mathrm{p}<0.05$, Mann-Whitney $U$ test between groups 1 and 2 .

${ }^{b)} p<0.05$, $p$-value was derived from repeated measures ANOVA for effect of time. 
linear hyperechoic strands that corresponded to fibroadipose septa (perimysium) and normal surrounding tissue.

RTS was performed as a color-coded graphic representation of relative stiffness of the structures within the selected ROI, such that purple indicated soft, green, and yellow indicated intermediate stiffness and red indicated stiff. An appropriate degree of natural compression was determined by manual adjustment, such that perimysium appeared predominantly yellow to red on RTS. The color-coded RTS image was depicted on the left side of the screen, while the ultrasound image was displayed on the right side. Our RTS protocol included standardized color encoding, and the same color scale was used for all the children.

The recorded RTS images were replayed in order to select the best representative image, which was selected based on an adequate depiction of tissue structure and consistent reproduction of the scanned images. Further, the color pattern of the recorded images was quantitatively analyzed on a personal computer using Image J software (National Institutes of Health, Bethesda, MD, USA) by another physiatrist. The color histogram represented the number of pixels that have colors in each of the fixed list of color ranges. The median red pixel intensity (RPI) within the ROI was calculated, with a high value derived from a high color intensity. RTS was performed
(A)

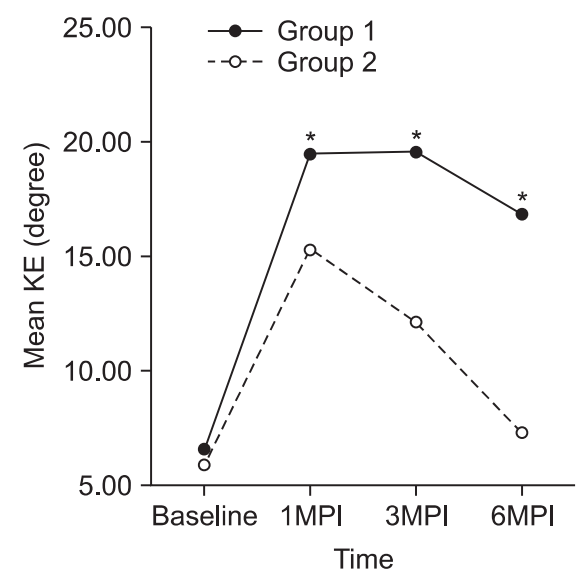

(D)

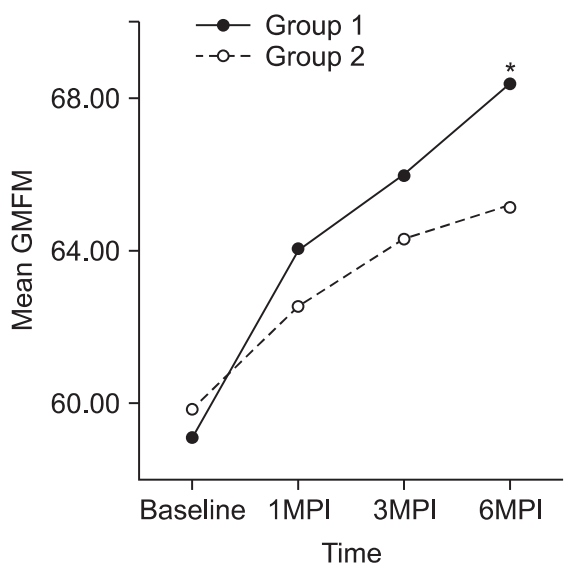

B

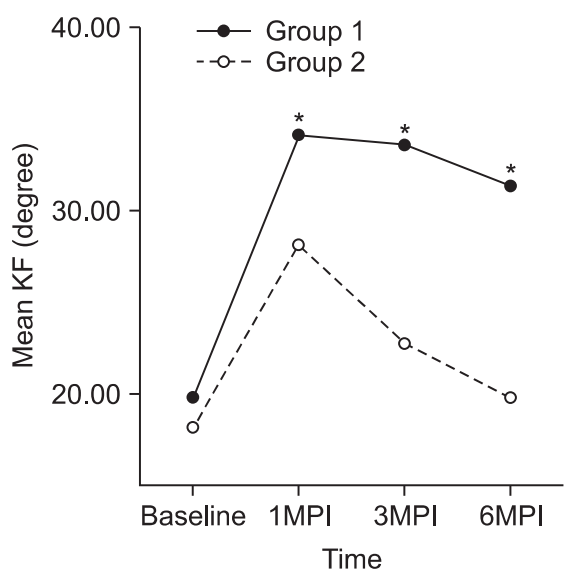

(E)

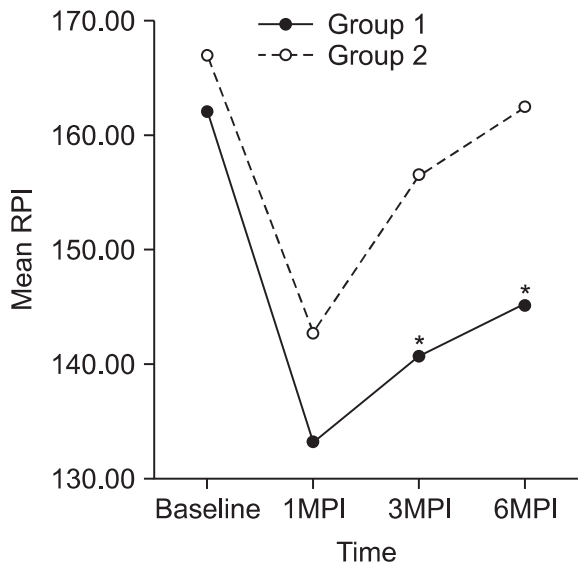

(C)

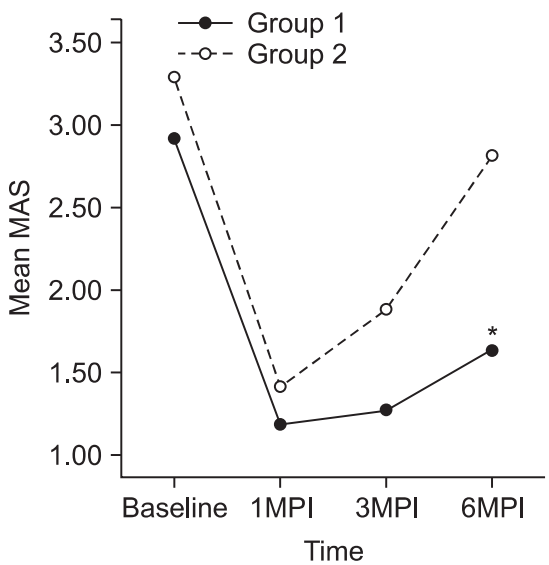

Fig. 1. Sequential changes in clinical and real-time sonoelastographic parameters at each month post-injection (MPI). In group 1 (under 5 years of age), there were significant differences in (A) passive range of motion of ankle dorsiflexion with knee extension (KE), (B) passive range of motion of ankle dorsiflexion with knee flexion (KF), (C) Modified Ashworth Scale (MAS), (D) Gross Motor Function Measure (GMFM), and (E) red pixel intensity (RPI) compared with those in group 2 (over 5 years of age). *p<0.05. 
twice, and a representative image was taken in each scan to check the intra-rater reliability of the measurements.

\section{Statistical analysis}

Statistical analysis was performed using SPSS ver. 19.0 (IBM Corporation, Armonk, NY, USA), with the level of significance set at $<0.05$. Fisher exact test was used for

Table 2. Comparison of changes in MAS at baseline and post-injection between group 1 and group 2

\begin{tabular}{ccc}
\hline & \multicolumn{2}{c}{ MAS } \\
\cline { 2 - 3 } & Group 1 $(\mathbf{n}=\mathbf{1 1})$ & Group 2 $(\mathbf{n}=15)$ \\
\hline Baseline & $2.9 \pm 0.5$ & $3.2 \pm 0.4$ \\
1MPI & $1.1 \pm 0.4^{\mathrm{b})}$ & $1.4 \pm 0.6^{\mathrm{b})}$ \\
$\Delta$ & $1.7 \pm 0.4$ & $1.8 \pm 0.3$ \\
3 MPI & $1.2 \pm 0.4^{\mathrm{b})}$ & $1.8 \pm 0.5^{\mathrm{b})}$ \\
$\Delta$ & $1.6 \pm 0.5$ & $1.4 \pm 0.7$ \\
6 MPI & $\left.1.6 \pm 0.5^{\mathrm{a}, \mathrm{b}}\right)$ & $2.8 \pm 0.4^{\mathrm{b})}$ \\
$\Delta$ & $1.2 \pm 0.4^{\mathrm{a})}$ & $0.4 \pm 0.6$ \\
\hline
\end{tabular}

Values are presented as mean \pm standard deviation.

MAS, Modified Ashworth Scale; MPI, month post-injection; $\Delta$, difference in the mean degrees between baseline and at each month post-injection; group 1 , under 5 years of age; group 2, over 5 years of age.

${ }^{a)} \mathrm{p}<0.05$, Mann-Whitney $U$ test between groups 1 and 2 .

${ }^{b)} \mathrm{p}<0.05, \mathrm{p}$-value was derived from repeated measures ANOVA for effect of time.

Table 3. Comparison of changes of GMFM at baseline and post-injection between group 1 and group 2

\begin{tabular}{ccc}
\hline & \multicolumn{2}{c}{ GMFM } \\
\cline { 2 - 3 } & Group 1 (n=7) & Group 2 (n=9) \\
\hline Baseline & $59.2 \pm 18.1$ & $59.9 \pm 18.8$ \\
1MPI & $64.1 \pm 19.6$ & $62.5 \pm 18.5$ \\
$\Delta$ & $4.9 \pm 4.1$ & $2.5 \pm 2.6$ \\
3MPI & $66.2 \pm 18.9$ & $64.3 \pm 16.5$ \\
$\Delta$ & $6.9 \pm 3.2$ & $4.4 \pm 4.2$ \\
6 MPI & $68.4 \pm 19.2$ & $65.2 \pm 17.1$ \\
$\Delta$ & $9.2 \pm 2.8^{\text {a) }}$ & $5.2 \pm 4.2$ \\
\hline
\end{tabular}

Values are presented as mean \pm standard deviation.

GMFM, Gross Motor Function Measure; MPI, month post-injection; $\Delta$, difference in the mean degrees between baseline and at each month post-injection; group 1 , under 5 years of age; group 2, over 5 years of age.

a) $\mathrm{p}<0.05$, Mann-Whitney $U$ test between groups 1 and 2 .

${ }^{\text {b) }} \mathrm{p}<0.05, p$-value was derived from repeated measures ANOVA for effect of time. the difference of gender, GMFCS level and topographical classification of $\mathrm{CP}$ between two groups at baseline. Within-group changes in measured parameters including PROM of ankle dorsiflexion, MAS of ankle plantar flexor, GMFM and RPI were analyzed with a two-factor repeated measures analysis of variance from baseline through 1-, 3-, and 6-month post-injections. Intergroup differences with respect to measured parameters were analyzed using the Mann-Whitney U test. The interclass correlation coefficient was used to examine the intra-rater reliability of repeated RPI measurements.

\section{RESULTS}

There were no significant differences in the gender, topographical classification and measured parameters including GMFCS level, PROM of ankle dorsiflexion, MAS of the ankle plantar flexor, GMFM and median RPI of the affected medial GCM between the two groups at baseline.

In both groups, the PROM of ankle dorsiflexion with knee extension and flexion significantly increased until 6-month post-injection. However, the change of PROM of ankle dorsiflexion in group 1 was significantly greater than that in group 2, until 6-month post-injection (Table 1; Fig. 1A, B). The mean MAS of the ankle plantar flexor was significantly decreased until 6-month post-injection in both groups (Table 2). The mean change in the MAS of

Table 4. Comparison of changes of RPI at baseline and post-injection between group 1 and group 2

\begin{tabular}{ccc}
\hline & \multicolumn{2}{c}{ RPI } \\
\cline { 2 - 3 } & Group 1 (n=11) & Group 2 (n=15) \\
\hline Baseline & $161.8 \pm 10.7$ & $166.6 \pm 10.3$ \\
1 MPI & $133.2 \pm 9.0^{\mathrm{b})}$ & $142.7 \pm 7.6^{\mathrm{b})}$ \\
$\Delta$ & $28.5 \pm 9.2$ & $23.9 \pm 5.8$ \\
3 MPI & $140.7 \pm 8.5^{\mathrm{a}, \mathrm{b}}$ & $156.3 \pm 8.4^{\mathrm{b})}$ \\
$\Delta$ & $21.0 \pm 0.3^{\mathrm{a})}$ & $10.3 \pm 6.9$ \\
6 MPI & $145.1 \pm 7.1^{\mathrm{a}, \mathrm{b}}$ & $162.3 \pm 11.9^{\mathrm{b})}$ \\
$\Delta$ & $16.6 \pm 9.1^{\mathrm{a})}$ & $4.3 \pm 8.3$ \\
\hline
\end{tabular}

Values are presented as mean \pm standard deviation.

RPI, red pixel intensity; MPI, month post-injection; $\Delta$, difference in the mean degrees between baseline and at each month post-injection; group 1, under 5 years of age; group 2, over 5 years of age.

${ }^{a)} \mathrm{p}<0.05$, Mann-Whitney $U$ test between groups 1 and 2 . ${ }^{b)} \mathrm{p}<0.05, \mathrm{p}$-value was derived from repeated measures ANOVA for effect of time. 
A
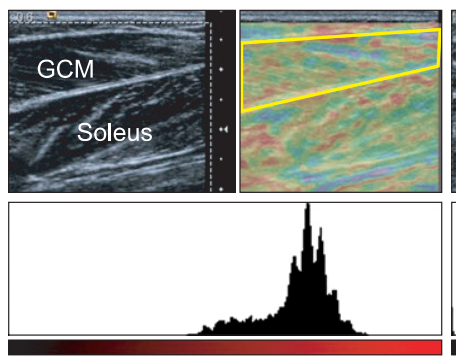

rMean: $169.70 \quad$ rSD: $20.52 \quad$ rMode: 17
B

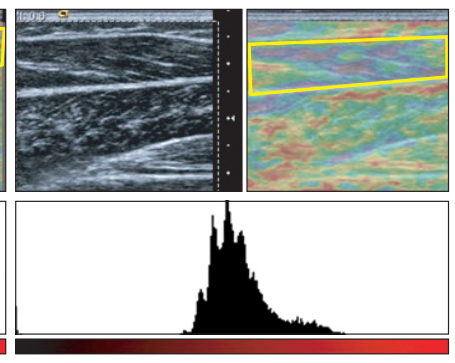

C

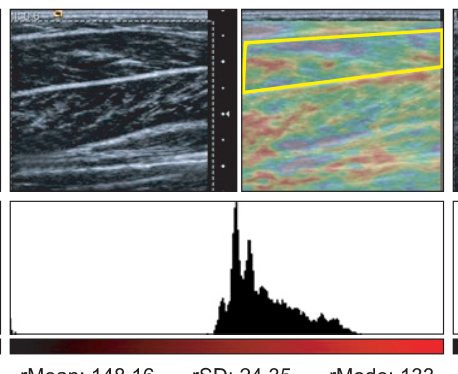

D

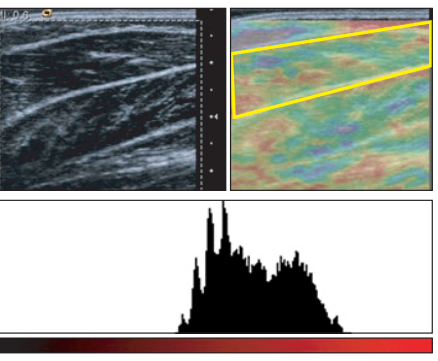

Fig. 2. Longitudinal real-time sonoelastography (RTS) image and color histogram of affected medial gastrocnemius muscle (GCM) in a 4-year-old boy. Yellow polygons and freehand selections depict regions of interest for quantitative analysis of the medial GCM on color histogram. (A) RTS image showed predominantly red color in medial GCM, and the median RPI of medial GCM was 169.7 at baseline. Follow-up RTS image showed purple to yellow color at posttreatment, and the median RPI was 130.9, 148.1, and 150.0 at 1 month (B), 3 months (C), and 6 months (D) post-treatment, respectively.
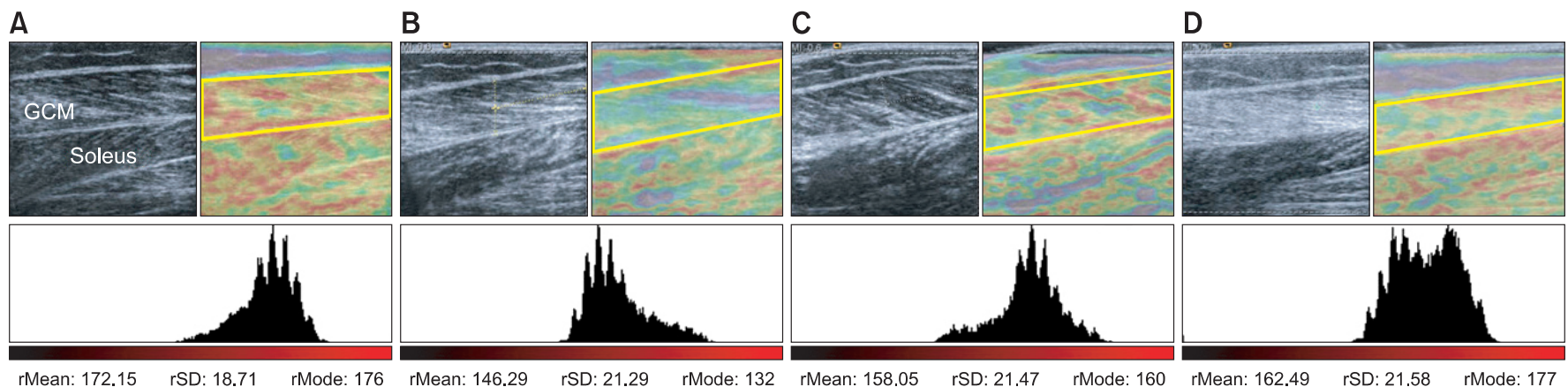

Fig. 3. Longitudinal real-time sonoelastography (RTS) image and color histogram of affected medial gastrocnemius muscle (GCM) in an 8-year-old boy. Yellow polygons and freehand selections depict regions of interest for quantitative analysis of the medial GCM on color histogram. (A) RTS image showed predominantly red color in medial GCM, and the median RPI of medial GCM was 172.1 at baseline. (B) RTS image showed purple to green color, and the median RPI was 146.2 at 1-month post-treatment. (C, D) RTS image showed green, yellow to red, and the median RPI was 158.0 and 162.4 at 3 and 6 months post-treatment, respectively.

the ankle plantar flexor between baseline and 6-month post-injection in group 1 was greater than that in group 2 (Table 2, Fig. 1C). However, there was no significant difference in the mean change in the MAS of the ankle plantar flexor between baseline and 1- and 3-month post-injections in either group (Table 2). There was no significant difference in the mean GMFM at 1-, 3-, and 6-month post-injections compared with baseline in both groups (Table 3 ). However, the mean changes in the GMFM between baseline and 6-month post-injection were greater in group 1 than that in group 2 (Table 3, Fig. 1D). The median RPI of the medial GCM was significantly decreased until 6-month post-injection in both groups. However, the mean changes in the median RPI of the medial GCM between baseline and 3- and 6-month post- injections in group 1 were greater than those in group 2 (Table 4; Figs. 1E, 2, 3).

The interclass correlation coefficients of repeated RPI measurements in groups 1 and 2 were 0.901 and 0.911 at baseline, and 0.905 and 0.902 at 1 -month, 0.915 and 0.907 at 3 -month, and 0.912 and 0.917 at 6 -month postinjection, respectively.

\section{DISCUSSION}

Our study demonstrated a significant difference in spasticity change in the ankle plantar flexor after BTA injection based on age in children with spastic CP. The mean MAS of the ankle plantar flexor significantly decreased at 1- and 3-month post-injections compared to that at 
baseline, in both groups. There was no significant difference in the mean change in the MAS of the ankle plantar flexor between baseline and 1- and 3-month post-injections between both groups. However, the mean change in the MAS of the ankle plantar flexor between baseline and 6 -month post-injection in children under 5 years of age was significantly greater than that in children over 5 years of age. These findings may be explained as follows. Because both groups received the same dose of BTA according to weight, the similar degrees of MAS reduction at 1- and 3-month post-injections in both groups were caused by the neuronal action of BTA, which is based on interference with the release of acetylcholine in the presynaptic terminals. It is maintained for approximately 90 days, and is dependent on the injection doses $[13,16]$. In addition to the reflex-induced neuronal component of passive stretch, non-neuronal components also contribute to resistance in passive stretch. Clinically assessed spasticity may rather be caused by non-neuronal changes in the spastic muscle and other tissues than by neuronal changes. These non-neuronal changes happen in both contractile and non-contractile tissues, such as stiffness of muscle fibers, loss of sarcomeres, changes in tendons and collagen tissue, and change in mechanical musclefiber properties [17]. In clinical practice, the MAS is probably the most commonly used test for the grading of spasticity and mainly measures the neuronal component of spasticity [18]. Therefore, neural component of spasticity plays an important role in reduction spasticity during 3-month post-injection. The difference of the mean change in the MAS at 6-month post-injection between groups may be related to the non-neuronal component of spasticity, which is caused by intrinsic muscle stiffness. When the block of neuronal component of spasticity by BTA injection wears off after 3-month post-injection [19], non-neuronal component of spasticity may play a major role in assessment of spasticity using MAS. In our study, the median RPI of the medial GCM on RTS significantly decreased in both groups until 6-month post-injection. However, the mean change in the median RPI between baseline and 3- and 6-month post-injections in children under 5 years of age was significantly greater than that for over 5 years of age. This difference may be attributed to alterations in passive muscle properties that are influenced by the age-related increase in non-reducible collagen content in the spastic muscle [13]. Because RTS indirectly assesses the biomechanical component of the spastic muscle, non-reducible collagen content in children over 5 years of age may be much higher than that in children under 5 years of age. Therefore, the muscular components may contribute to the difference of decreased spasticity, as measured by the MAS at 6-months post-injection

In our study, the mean change of PROM of ankle dorsiflexion in children under 5 years of age was significantly greater than that in children over 5 years of age at 1-, 3-, and 6-month post-injections. There is a possible explanation for the greater change of PROM of ankle dorsiflexion after BTA injection in children under 5 years of age. Resistance to passive motion is the sum of the reflex muscle activity and non-neuronal changes. However, non-neuronal changes in spastic muscle mainly contribute to the resistance to joint motion. Restricted joint range of motion due to structural alterations of spastic muscle is influenced by the age-related increase of nonreducible collagen content in spastic muscle. In comparison with a normal muscle cell, a spastic muscle cell has a short resting sarcomere and an increased elastic modulus, implying dramatic remodeling of intra- and extracellular structural components, such as titin and collagen [20]. Collagen has an important physiological role in the development of muscle stiffness [21]. In children with spastic $\mathrm{CP}$, non-reducible collagen content in the spastic muscle increases with age, because inactivity due to muscle spasticity leads to muscle weakness, disused muscle atrophy and muscle shortening [13]. Rehabilitation treatment after BTA injection may have a differential effect on the reorganization of collagen bundles in intramuscular connective tissue of the ankle plantar flexor, according to the age of children with spastic CP [22]. Therefore, the change of PROM and RPI due to a decrease of intrinsic muscle stiffness in children under 5 years of age was greater than that in children over 5 years of age, from the early period after intensive rehabilitation treatment for 4 weeks

The mean change in the GMFM between baseline and 6 -month post-injection in children under 5 years of age was greater than that in children over 5 years of age. The improvement in gross motor function in both groups was influenced by the natural maturational development of children, combined with rehabilitation treatment and BTA injection. A previous study [23] claimed that in terms 
of the rate of development, children in GMFCS level III were expected to have reached about $90 \%$ of their potential by about age of 3.7 years. Other studies $[24,25]$ showed that the majority of children at GMFCS level III reached their top GMFM at the age of 7-8 years. In our study, eight of the nine children in group 2 were GMFCS level III, and the mean age was approximately 9.2 years. Therefore, a greater improvement in gross motor function was observed in children under 5 years of age. Our results agree with those of an earlier study [15], which has reported that younger children with $\mathrm{CP}$ are likely to show greater effects after BTA injection.

The intra-rater reliability of RPI measurement in the medial GCM on RTS was good in our study. These results are in accordance with those obtained by Nordez et al. [26], which showed a good reliability of local muscle stiffness at rest, using transient sonoelastography in vivo.

This study has several limitations. First, it is not advisable to generalize our results in children with spastic $\mathrm{CP}$, because our sample size is small. Further studies with a large sample size and longer follow-up are necessary to establish the validity of our results. Second, double blinding, as well as blinding of the children and examiner, was not feasible because of the nature of the treatment method. Third, we could not evaluate whether inter-rater reliability of RPI measurement. RTS is operator dependent, requires long-term experience and has a technical problem in image reproducibility due to instability of the applied pressure using the free-hand technique [8]. Last, we did not contemplate the reliability of the MAS of the ankle plantar flexor in children with spastic CP. A previous study [27] has claimed that the MAS has an acceptable inter-rater reliability for testing upper limb spasticity, but not for testing lower limb spasticity.

In conclusion, our pilot study demonstrated the different changes in the spasticity of the ankle plantar flexor after BTA injection based on age. Therefore, we suggest that age may be considered when determining BTA injection in children with spastic CP.

\section{CONFLICT OF INTEREST}

No potential conflict of interest relevant to this article was reported.

\section{ACKNOWLEDGMENTS}

We appreciate Medytox Inc. for their financial contribution to the study. This work was supported by the Basic Science Research Program through the National Research Foundation of Korea (NRF) funded by the Ministry of Education, Science and Technology (2012-0003178).

\section{REFERENCES}

1. Bohannon RW, Smith MB. Interrater reliability of a modified Ashworth scale of muscle spasticity. Phys Ther 1987;67:206-7.

2. Gracies JM, Burke K, Clegg NJ, Browne R, Rushing C, Fehlings D, et al. Reliability of the Tardieu Scale for assessing spasticity in children with cerebral palsy. Arch Phys Med Rehabil 2010;91:421-8.

3. Dietz V, Sinkjaer T. Spastic movement disorder: impaired reflex function and altered muscle mechanics. Lancet Neurol 2007;6:725-33.

4. Booth CM, Cortina-Borja MJ, Theologis TN. Collagen accumulation in muscles of children with cerebral palsy and correlation with severity of spasticity. Dev Med Child Neurol 2001;43:314-20.

5. Kim K, Shin HI, Kwon BS, Kim SJ, Jung IY, Bang MS. Neuronox versus BOTOX for spastic equinus gait in children with cerebral palsy: a randomized, doubleblinded, controlled multicentre clinical trial. Dev Med Child Neurol 2011;53:239-44.

6. Ade-Hall RA, Moore AP. Botulinum toxin type A in the treatment of lower limb spasticity in cerebral palsy. Cochrane Database Syst Rev 2000;(2):CD001408.

7. Cosgrove AP, Corry IS, Graham HK. Botulinum toxin in the management of the lower limb in cerebral palsy. Dev Med Child Neurol 1994;36:386-96.

8. Borodic GE, Ferrante R, Pearce LB, Smith K. Histologic assessment of dose-related diffusion and muscle fiber response after therapeutic botulinum A toxin injections. Mov Disord 1994;9:31-9.

9. Park GY, Kwon DR. Application of real-time sonoelastography in musculoskeletal diseases related to physical medicine and rehabilitation. Am J Phys Med Rehabil 2011;90:875-86.

10. Kwon DR, Park GY, Lee SU, Chung I. Spastic cerebral palsy in children: dynamic sonoelastographic findings of medial gastrocnemius. Radiology 2012;263:794-801. 
11. Park GY, Kwon DR. Sonoelastographic evaluation of medial gastrocnemius muscles intrinsic stiffness after rehabilitation therapy with botulinum toxin a injection in spastic cerebral palsy. Arch Phys Med Rehabil 2012;93:2085-9.

12. Kwon DR, Park GY, Kwon JG. The change of intrinsic stiffness in gastrocnemius after intensive rehabilitation with botulinum toxin A injection in spastic diplegic cerebral palsy. Ann Rehabil Med 2012;36:400-3.

13. de Paiva A, Meunier FA, Molgo J, Aoki KR, Dolly JO. Functional repair of motor endplates after botulinum neurotoxin type A poisoning: biphasic switch of synaptic activity between nerve sprouts and their parent terminals. Proc Natl Acad Sci U S A 1999;96:3200-5.

14. Pierce SR, Prosser LA, Lauer RT. Relationship between age and spasticity in children with diplegic cerebral palsy. Arch Phys Med Rehabil 2010;91:448-51.

15. Pascual-Pascual SI, Pascual-Castroviejo I, Ruiz PJ. Treating spastic equinus foot from cerebral palsy with botulinum toxin type A: what factors influence the results?: an analysis of 189 consecutive cases. Am J Phys Med Rehabil 2011;90:554-63.

16. Wang Y, Gao B. A dose-response relationship research on botulinum toxin type A local intramuscular injections of lower extremity spasticity in children with cerebral palsy. Childs Nerv Syst 2008;24:545-7.

17. Dietz V, Trippel M, Berger W. Reflex activity and muscle tone during elbow movements in patients with spastic paresis. Ann Neurol 1991;30:767-79.

18. Sommerfeld DK, Gripenstedt U, Welmer AK. Spasticity after stroke: an overview of prevalence, test instruments, and treatments. Am J Phys Med Rehabil 2012;91:814-20.

19.Zimmerman SD, McCormick RJ, Vadlamudi RK, Thomas DP. Age and training alter collagen character- istics in fast- and slow-twitch rat limb muscle. J Appl Physiol (1985) 1993;75:1670-4.

20. Friden J, Lieber RL. Spastic muscle cells are shorter and stiffer than normal cells. Muscle Nerve 2003;27:157-64.

21. Feit H, Kawai M, Mostafapour AS. Increased resistance of the collagen in avian dystrophic muscle to collagenolytic attack: evidence for increased crosslinking. Muscle Nerve 1989;12:476-85.

22. Coutinho EL, DeLuca C, Salvini TF, Vidal BC. Bouts of passive stretching after immobilization of the rat soleus muscle increase collagen macromolecular organization and muscle fiber area. Connect Tissue Res 2006;47:278-86.

23. Rosenbaum PL, Walter SD, Hanna SE, Palisano RJ, Russell DJ, Raina P, et al. Prognosis for gross motor function in cerebral palsy: creation of motor development curves. JAMA 2002;288:1357-63.

24. Harries N, Kassirer M, Amichai T, Lahat E. Changes over years in gross motor function of 3-8 year old children with cerebral palsy: using the Gross Motor Function Measure (GMFM-88). Isr Med Assoc J 2004;6:40811.

25. Beckung E, Carlsson G, Carlsdotter S, Uvebrant P. The natural history of gross motor development in children with cerebral palsy aged 1 to 15 years. Dev Med Child Neurol 2007;49:751-6.

26. Nordez A, Gennisson JL, Casari P, Catheline S, Cornu C. Characterization of muscle belly elastic properties during passive stretching using transient elastography. J Biomech 2008;41:2305-11.

27. Mutlu A, Livanelioglu A, Gunel MK. Reliability of Ashworth and Modified Ashworth scales in children with spastic cerebral palsy. BMC Musculoskelet Disord 2008;9:44. 